\title{
DINÂMICA DAS PARTÍCULAS DE SOJA EM TAMBOR ROTATIVO DE BANCADA EMPREGANDO O MÉTODO DE ELEMENTOS DISCRETOS
}

\author{
N. P. ALMEIDA ${ }^{1}$, F. P. TAVARES ${ }^{1}$, K. G. SANTOS ${ }^{1 *}$ \\ ${ }^{1}$ Universidade Federal do Triângulo Mineiro, Departamento de Engenharia Química \\ e-mail: kassiagsantos@gmail.com
}

\begin{abstract}
RESUMO
São muitos os processos industriais que envolvem operações com partículas. A descrição matemática do escoamento de sistemas multifásicos granulares é uma ferramenta importante para auxiliar na compreensão do processo de transporte de sólidos. O Método de Elementos Discretos (DEM) é uma técnica que representa de maneira eficiente o comportamento discreto em sistemas com partículas de baixa densidade e esfericidade, como a semente de soja. Este trabalho avaliou simulações de um tambor rotativo de bancada contendo soja como partícula analisada. As simulações foram feitas com diferentes combinações de valores de coeficientes de restituição, fricção e elasticidade, que são parâmetros para o modelo linear "mola-amortecedor". Através da comparação dessas simulações com dados experimentais de ângulo dinâmico de repouso foi possível calibrar os parâmetros de colisão para a semente de soja.
\end{abstract}

Palavras-chave: Ângulo de repouso. DEM. Parâmetros de colisão. Soja.

\section{INTRODUÇÃO}

Existem muitos processos industriais que envolvem sistemas particulados. A descrição matemática do escoamento de sistemas multifásicos granulares é atualmente uma ferramenta com grande potencial para auxiliar na compreensão do processo de transporte de sólidos. Essa modelagem é complexa e pode ser efetuada por diferentes abordagens (Lagrangeana e a Euleriana), que apresentam vantagens e limitações (SANTOS et al., 2009).

O crescente desenvolvimento dos computadores abriu novas possibilidades para a abordagem de problemas que exigem cálculos e soluções para os mais completos modelos matemáticos, como é o caso das simulações pela técnica da fluidodinâmica computacional (CFD). A utilização de CFD para as simulações da fluidodinâmica e secagem é de fundamental importância para a compreensão dos fenômenos envolvidos (GRACE E TAGHIPOUR, 2004), contribuindo assim para a otimização do processo, tendo em vista os aspectos energéticos, sem perder de vista a qualidade do produto final.

A técnica de simulação CFD tem auxiliado a compreensão dos fenômenos inerentes a diversos processos como: separação sólido-fluido em hidrociclones convencionais e filtrantes (VIEIRA, 2006); otimização do recobrimento de sementes de soja em leito de jorro (DUARTE, 2006); escoamento laminar de fluidos não Newtonianos em seções anulares (PEREIRA, 2006); extração mecânica da bixina a partir de 
urucum em leito de jorro (CUNHA, 2008); pirólise de biomassa em reatores de leito de jorro e leito fluidizado (SANTOS, 2011); secadores em leito de jorro e em tambor rotativo (SILVÉRIO, 2012).

Estudos fundamentais sobre a fluidodinâmica do leito de jorro e fluidizado têm possibilitado uma análise aprofundada do efeito das principais variáveis envolvidas no processo, visando superar algumas importantes limitações do leito de jorro e encontrar as configurações ótimas para as diversas aplicações deste equipamento.

No caso de leitos móveis, a modelagem utilizando a aproximação Euler-Euler, que trata as fases como contínuas e interpenetrantes, apresenta boa predição para sistemas particulados densos, principalmente com partículas esféricas e de maior densidade. Os resultados apresentados por Santos (2011) mostraram que a abordagem Euleriana reproduz adequadamente a segregação de misturas contendo partículas de mesmo material (mesma densidade) e diâmetros diferentes. No entanto, os testes de simulação CFD realizados com matérias de densidades diferentes revelaram que a abordagem não é a mais adequada, principalmente quando se trabalha com biomassa como material particulado.

De acordo com Gao e Sun (2011), a maior falha da abordagem Euler-Euler aplicada a sistemas particulados é que ele não captura as características individuais das partículas sólidas quanto à sua forma e tamanho e não pode identificar efetivamente a influência dessas características na fluidodinâmica da partícula. Isso ocorre principalmente para sistemas com materiais particulados heterogêneos, com baixa esfericidade e baixa densidade (o que implica em choques menos elásticos), como é o caso das biomassas em geral.

Segundo Souza (2010), um dos maiores desafios da modelagem de sistemas granulares é ainda modelar todas as características desse complexo escoamento, principalmente se tratando de sistemas densos. Para uma simulação a nível microscópico, cada partícula deve ser considerada como um sistema que tem seu movimento descrito pelas Leis de Newton. Nesse aspecto, novas metodologias computacionais têm sido desenvolvidas, como o Método de Elementos Discretos (DEM).

Até bem recentemente, o Método de Elementos discretos era limitado a sistemas com fase particulada dispersa (fração volumétrica inferior a 10\%), ou seja, o movimento das partículas não influência o escoamento do fluido e este determina a trajetória da partícula. Por isso, este modelo era inadequado para a modelagem de misturas líquido-líquido ou para sistemas em que a fração volumétrica da fase secundária é significante, que é o caso do leito de jorro, leito fixo e leito fluidizado.

No entanto, esta limitação da abordagem Euleriana-Lagrangeana foi praticamente vencida. Já se encontram na literatura alguns trabalhos de simulação discreta de partículas para prever a fluidodinâmica de equipamentos com fase densa, como leitos fixo, de jorro, leito fluidizado, hidrociclones com fase densa, secadores, dentre outros. $\mathrm{O}$ acoplamento entre CFD e métodos de elementos discretos tem se mostrado uma técnica promissora e robusta, capaz de reproduzir diferentes regimes de escoamento.

O Método de Elementos Discretos é visado como uma boa alternativa para a modelagem de sistemas particulados densos, pois contabiliza o efeito do tamanho e da forma real da partícula, além de permitir uma modelagem detalhada da colisão entre as partículas com outras partículas e com a parede do recipiente.

Nota-se que os valores encontrados para os parâmetros dos modelos nos trabalhos de simulação DEM variam significativamente, pois cada estudo foi concebido para equipamentos distintos e representa o escoamento de materiais diferentes os quais podem conduzir diferentes respostas de colisões partícula-partícula e partícula-parede. 
Sendo assim, a abordagem DEM necessita de uma calibração prévia dos parâmetros do modelo de contato entre partículas, a fim de que o choque destas na simulação seja similar ao que ocorre experimentalmente. Para tal, empregam-se testes experimentais simples, como, por exemplo, os ângulos de repouso estático e dinâmico das partículas.

O ângulo de repouso estático é definido como o ângulo interno entre a superfície lateral da pilha e o plano horizontal e é uma característica própria do material particulado, que é função da densidade da partícula, área superficial e forma, além do coeficiente de atrito do material.

O ângulo de repouso dinâmico é referente ao movimento superficial do produto como se estivesse em uma mesa inclinada ou tambor rotativo e sua medida caracteriza a escoabilidade do material. É o ângulo formado entre o material inclinado e o plano horizontal. Este ângulo é uma função do tipo de material e suas propriedades, bem como da velocidade angular de rotação do cilindro. A Figura 1 mostra um exemplo de medida dos ângulos de repouso estático e dinâmico.

Figura 1 - Ângulos de repouso: (a) estático (b) dinâmico.

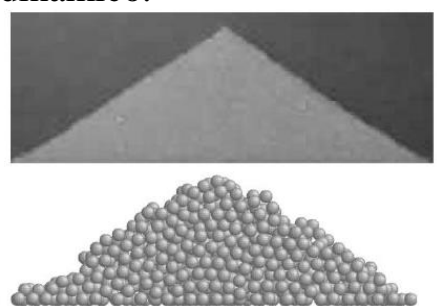

(a)

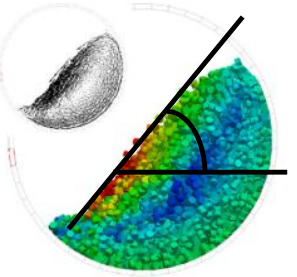

(b)
Fontes: (a) ZHOU et al.(2002) (b) WACHS et al. (2012).

Walton e Braun (1993) investigaram o efeito da forma da partícula no ângulo de repouso dinâmico e estático usando DEM, empregando esferas e cubos. Eles concluíram que o ângulos de repouso estático e dinâmico aumentam com o aumento da nãoesfericidade da partícula.

O conhecimento da dinâmica das partículas em equipamentos como secador em tambor rotativo, leitos fixo e leitos móveis; geralmente aplicados para secagem, recobrimento de partículas, como reatores de craqueamento catalítico ou pirólise; pode favorecer o projeto e otimização de diversos processos nesses equipamentos, desde que sejam calibrados os parâmetros de força de contato entre as partículas.

Silvério (2012) estudou a secagem de fertilizantes em um tambor rotativo convencional, que continha suspensores em diversas posições angulares. Neste equipamento, a quantidade de sólidos contida em cada suspensor pode ser calculada em função da sua geometria, da posição angular no interior do tambor e do ângulo dinâmico de repouso formado entre os sólidos e a superfície horizontal. O início do cascateamento, essencial para promover o transporte dos sólidos, se dá quando o ângulo da superfície de sólido em relação ao plano horizontal ultrapassa o valor do ângulo de repouso naquela posição angular.

A literatura apresenta vários modelos de colisão entre as partículas: Modelo Linear de Mola (spring), Modelo Linear MolaAmortecedor (spring-dashpot), Modelo de Hertz-Mindlin (com ou sem a adição da Lei de Fricção de Coulomb), dentre outros. Di Renzo (2004) avaliou três diferentes modelos para forças de contato e constatou que o modelo linear "mola-amortecedor" é eficiente na predição das forças de contato entre as partículas.

No entanto, os dados sobre a colisão de partículas disponíveis na literatura cobrem apenas uma pequena quantidade de materiais, sendo necessária a calibração individual de cada tipo de material com o qual deseja-se trabalhar.

Sendo assim, o objetivo deste trabalho foi realizar a calibração dos parâmetros do modelo de contato entre as partículas de soja (Modelo Mola-Amortecedor) empregando o ângulo de repouso, para futura simulação da dinâmica dessas mesmas partículas em diferentes equipamentos, como leitos de jorro e secadores de leito deslizante. 
Para tal, foi montado um planejamento composto central para avaliar o efeito de cada parâmetro do modelo na resposta de ângulo de repouso dado pela simulação, a fim de encontrar quais os parâmetros afetam significativamente o ângulo simulado. Os parâmetros do modelo Mola-amortecedor avaliados foram os o coeficientes de restituição $(\eta)$, de fricção $(\mu)$ e o coeficiente de elasticidade ou mola (k). Os dados simulados empregando DEM foram comparados com os valores obtidos experimentalmente, a fim de estabelecer o conjunto de parâmetros que representam a colisão da partícula em questão.

\section{MÉTODO DE ELEMENTOS DISCRETOS (DEM)}

O software FLUENT® 14.0, uma vez que se opta pela utilização do modelo Euleriano, além de resolver as equações de transporte para a fase contínua, permite simular uma fase discreta segundo uma abordagem Lagrangeana que utiliza o DEM (Discret Element Method). Esta segunda fase consiste em partículas esféricas presentes na fase contínua. $\mathrm{O}$ modelo calcula as trajetórias das fases discretas e contínuas. $\mathrm{O}$ acoplamento entre as fases e o seu impacto sobre ambas as trajetórias da fase discreta e o fluxo de fase contínua também é incluído nos cálculos (SILVÉRIO, 2012).

O modelo utilizado resolve para a fase fluida a Equação 1 da Continuidade e a Equação 2 do Movimento.

$\frac{\partial \rho}{\partial t}+\nabla(\rho \vec{v})=0$

$\frac{\partial}{\partial t}(\rho \vec{v})+\nabla(\rho \vec{v} \vec{v})=-\nabla \rho+\nabla(\overline{\bar{\tau}})+\rho \vec{g}+\vec{F}$

A utilização de modelos DEM (Discret Element Method) permite simular partículas em movimento como pontos de massa em movimento. A partir da segunda lei de Newton, as equações diferenciais ordinárias que regem o movimento das partículas são representadas por (SILVÉRIO, 2012):

$$
\begin{aligned}
& m \frac{d \vec{v}_{p}}{d t}=\vec{F}_{a}+\vec{F}_{p}+\vec{F}_{m v}+\vec{F}_{g}+\vec{F}_{o} \\
& \vec{v}_{p}=\frac{d x}{d t}
\end{aligned}
$$

na qual $\vec{v}_{p}$ é velocidade da partícula, $x$ é a posição da partícula e $t$ é o tempo.

A implementação DEM é baseada no trabalho de Cundall e Strack (1979), e contabiliza as forças resultantes da colisão das partículas por meio da aproximação da "esfera macia".

As forças de colisão de partículas são determinadas pela deformação, que é medida como a sobreposição entre os pares de esferas, ou entre uma esfera e um limite. A Equação 3 é integrada ao longo do tempo para capturar a intersecção das partículas utilizando-se uma escala de tempo que é determinada pela rigidez dos materiais (SILVÉRIO, 2012).

As forças de contato entre as partículas $F_{i j}$, contabilizadas no termo $\vec{f}_{\text {perda }}$ da Equação 8, são representadas pelo modelo de colisão chamado "mola-amortecedor" (spring-dashpot model). Essas forças são decompostas nas direções normal e tangencial, representado na Figura 2.

Figura 2 - Modelo de forças de contato (Modelo Mola-amortecedor).

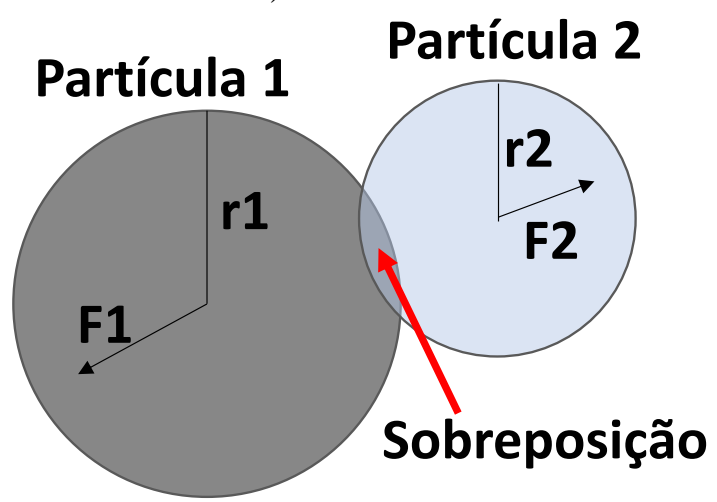

Fonte: Autor (2015)

Por sua vez, as componentes normal e tangencial da força de contato $F_{i j}$ são decompostas em forças elásticas ou de repulsão (chamadas de conservativas), responsáveis pelo movimento de translação, e 
forças de amortecimento (chamadas de dissipativas), responsáveis pelo movimento de rotação das partículas.

O valor da constante de elasticidade na força normal de contato entre partículas deve ser suficientemente alto para fazer duas partículas recuarem em uma colisão, fazendo uma sobreposição máxima menor que o raio da partícula, para uma alta velocidade relativa entre elas.

É possível estimar o valor da constante de elasticidade $k$ através da seguinte equação (CUNDALL e STRACK, 1979):

$k \geq \sqrt[3]{\frac{2 v_{p}^{2}}{3 \pi \varepsilon_{D}^{2}} d_{p}}$

na qual $d_{p}$ é o diâmetro da partícula $v_{p}$ é o módulo da velocidade relativa entre as duas partículas que estão colidindo, $\varepsilon_{D}$ é a fração do diâmetro que se permite sobrepor, $\pi \sqrt{m / k}$ é o tempo de colisão avaliado e $m$ é a massa da partícula dada por $\left(\rho d_{p}^{3} \pi\right) / 6$.

Para a colisão elástica linear, o vetor unitário $\left(\vec{e}_{12}\right)$ é definido da partícula 1 para a partícula 2:

$\vec{e}_{12}=\frac{\left(x_{2}-x_{1}\right)}{\left\|x_{2}-x_{1}\right\|}$

na qual $x_{1}$ e $x_{2}$ representam as posições das partículas 1 e 2, respectivamente. A sobreposição $\delta$ (que durante o contato é menor do que zero) é definida como:

$\delta=\left\|x_{2}=x_{1}\right\|-\left(r_{1}+r_{2}\right)$

sendo que $r_{1}$ e $r_{2}$ representam os raios das partículas 1 e 2 .

Para o modelo de colisão "molaamortecedor", define-se uma constante como na lei de colisão elástica $k$, junto com um coeficiente de restituição para o termo amortecedor $(\eta)$. Nota-se que $0<\eta \leq 1$.

Para os cálculos das forças, as seguintes expressões são avaliadas:

$\vec{f}_{\text {perda }}=\sqrt{\pi^{2} \ln ^{2} \eta}$

$\mathrm{m}_{12}=\frac{\mathrm{m}_{1} \mathrm{~m}_{2}}{\mathrm{~m}_{1}+\mathrm{m}_{2}}$

$$
\begin{aligned}
& \mathrm{t}_{\mathrm{col}}=\mathrm{f}_{\text {perda }} \sqrt{\frac{\mathrm{m}_{12}}{\mathrm{k}}} \\
& \gamma=-2 \frac{\mathrm{m}_{12} \ln \eta}{\mathrm{t}_{\mathrm{col}}} \\
& \overrightarrow{\mathrm{v}}_{\mathrm{p} 12}=\overrightarrow{\mathrm{v}}_{\mathrm{p} 1}+\overrightarrow{\mathrm{v}}_{\mathrm{p} 2}
\end{aligned}
$$

na qual $\vec{f}_{\text {perda }}$ é um fator de redução ou perda de massa, $m_{1}$ e $m_{2}$ são as massas das partículas 1 e 2 , respectivamente, $m_{12}$ é a chamada "massa reduzida", $t_{c o l}$ é o tempo de colisão, e $\vec{v}_{p 1}$ e $\vec{v}_{p 2}$ são as velocidades das partículas 1 e 2 , respectivamente, $\vec{v}_{p 12}$ é a velocidade relativa entre as partículas 1 e 2 , e $\gamma$ é o coeficiente de amortecimento.

A força sobre uma partícula pode ser calculado como na Equação 13 (CUNDALL e STRACK, 1979):

$\vec{F}_{1}=\left(k \delta+\gamma\left(\vec{V}_{12} \vec{e}_{12}\right)\right) \vec{e}_{12}$

E de acordo com a $3^{\text {a }}$ Lei de Newton a força exercida na partícula 2 é:

$\vec{F}_{2}=\vec{F}_{1}$

A lei do coeficiente de fricção é baseada na equação de fricção de Coulomb $\vec{F}_{\text {fric }}$.

$\vec{F}_{\text {fric }}=\mu_{f} \vec{F}_{\text {normal }}$

na qual $\mu_{f}$ é o coeficiente de fricção e $\vec{F}_{\text {normal }}$ é a força normal à superfície. A direção da força de fricção é oposta ao movimento tangencial e pode ou não inibir o movimento tangencial relativo Silvério (2012). O coeficiente de fricção é uma função do módulo da velocidade tangencial relativa $\left(\vec{v}_{p r t}\right)$.

$$
\begin{aligned}
& \mu_{f}\left(\vec{v}_{p r}\right)= \mu_{f \text { perf }}+\left(\mu_{f \text { perf }}-\mu_{f \text { desl }}\right) \cdot \\
& \cdot\left(\frac{\vec{v}_{p r}}{\vec{v}_{p-\text { desl }}}-2\right)\left(\frac{\vec{v}_{p r}}{\vec{v}_{p-\text { desl }}}\right)
\end{aligned}
$$

$\vec{v}_{p-\text { desl }}<\vec{v}_{p r} \leq \vec{v}_{p-\text { lim }}$

em que $\mu_{f \text { perf }}$ é o coeficiente de atrito de perfuração; $\mu_{f \text { desl }}$ é o coeficiente de atrito de deslizamento; $\mu_{f \text { lim }}$ é o coeficiente de atrito de alta velocidade limite; $\vec{v}_{p \text { desl }}$ é a velocidade de deslizamento. 
Para velocidades mais baixas, $\mu \mathrm{f}$ é obtido por interpolação quadrática entre $\mu_{f \text { perf }}$ e $\mu_{f \text { desl }}, \vec{v}_{p \text {-lim }}$ é a velocidade limite, para velocidades mais altas, $\mu_{f}\left(\vec{v}_{p r}\right)$ aproxima-se de $\mu_{f \text { lim }}$.

\section{METODOLOGIA}

\subsection{Procedimento Experimental}

$\mathrm{O}$ experimento foi realizado em um cilindro rotativo de acrílico, com $0,1 \mathrm{~m}$ de diâmetro e $0,1 \mathrm{~m}$ de comprimento, operando a $25 \mathrm{rpm}$. A massa de semente de soja foi adicionada de modo que $50 \%$ do volume do tambor fosse preenchido.

O ângulo dinâmico de repouso foi obtido através de fotografias do experimento.

\subsection{Metodologia de Simulação}

Nas simulações, utilizou-se o software FLUENT a fim de se obter o ângulo dinâmico de repouso das partículas dentro e, assim, avaliar os parâmetros do Método dos Elementos Discretos (DEM).

A malha computacional tridimensional foi construída a partir do software GAMBIT®. A geometria do tambor simulado é idêntica à do experimental, com $0,1 \mathrm{~m}$ de diâmetro e $0,1 \mathrm{~m}$ de comprimento, composta por 445 células.

O conjunto de equações de conservação e constitutivas foi resolvido utilizando-se o método dos volumes finitos. $\mathrm{O}$ algoritmo SIMPLE foi utilizado para acoplar velocidade e pressão. Os resíduos utilizados foram menores que $1 \cdot 10^{-3}$. Na parede, considerou-se uma condição de contorno de não deslizamento. Para a discretização espacial utilizou-se o modelo QUICK e para a discretização temporal o modelo implícito de primeira ordem.

A fim de avaliar o efeito dos parâmetros do modelo DEM sobre o ângulo dinâmico de repouso das partículas de soja, as simulações foram definidas por meio de um planejamento composto central (PCC) ortogonal com 7 réplicas no ponto central. A Tabela 1 mostra os níveis adotados no planejamento e os respectivos valores das variáveis reais.

Tabela 1 - Níveis e faixas de valores das variáveis codificadas em estudo.

\begin{tabular}{lrcccc}
\hline & \multicolumn{5}{c}{ Níveis } \\
\cline { 2 - 6 } \multicolumn{1}{c}{ Variáveis } & $-1,575$ & -1 & 0 & 1 & 1,575 \\
\hline $\mathrm{k}[\mathrm{N} / \mathrm{m}]\left(\mathrm{X}_{1}\right)$ & 54,98 & 400 & 1000 & 1600 & 1945 \\
$\mu\left(\mathrm{X}_{2}\right)$ & 0,027 & 0,2 & 0,5 & 0,8 & 0,973 \\
$\eta\left(\mathrm{X}_{3}\right)$ & 0,027 & 0,2 & 0,5 & 0,8 & 0,973 \\
\hline
\end{tabular}

Fonte: Autor (2015)

Os valores dos parâmetros escolhidos tiveram como base a ampla faixa encontrada na revisão da literatura. Sendo assim, nem todas as combinações entre os parâmetros necessariamente conduzem a resultados fisicamente coerentes, mas pode contribuir para verificar seus efeitos e suas interações. Foi gerado um conjunto de imagens do interior do tambor em movimento para cada simulação do PCC, sendo que as condições adotadas nas simulações estão descritas na Tabela 2. Através destas simulações foi possível obter os ângulos de repouso dinâmicos médios para cada uma delas.

Tabela 2 - Condições adotadas nas simulações do secador rotatório convencional.

\begin{tabular}{ll} 
Time step fase contínua (s) & 0,001 \\
Time step fase discreta (s) & 0,0002 \\
Velocidade rotacional (rpm) & 25 \\
Número de partículas & 416 \\
Tempo simulado (s) & 12 \\
\hline
\end{tabular}

Fonte: Autor (2015).

Além de comparar os resultados do ângulo dinâmico de repouso obtido nas simulações aos dados experimentais, para a escolha dos parâmetros de simulação que melhor caracterizam o escoamento da soja em tambores, foi feita a comparação visual qualitativa entre as imagens obtidas durante o experimento e os perfis simulados de posição das partículas.

Também foi realizada uma análise de regressão múltipla para quantificar o efeito dos parâmetros do modelo sobre o ângulo 
simulado. Trabalhos da literatura empregam a metodologia de superfície de resposta, não apenas para planejar e analisar resultados experimentais, mas também simulações e processos de otimização (JOHNSTONE, 2010; SILVÉRIO, 2012).

\section{RESULTADOS E DISCUSSÕES}

Cada simulação permitiu analisar o ângulo de repouso dinâmico, em função dos três parâmetros estudados (coeficiente de restituição, coeficiente de fricção e coeficiente de elasticidade).

A Figura 3 mostra a comparação entre o ângulo dinâmico experimental das partículas de soja no interior do tambor rotativo com algum dos ângulos simulados no tempo de $12 \mathrm{~s}$.
De posse das imagens da Simulação 1 à Simulação 21, foi possível montar a Tabela 3 , em que estão dispostos os ângulos médios para cada análise do PCC.

Na Figura 4 estão dispostos os perfis de posição das partículas de soja para cada simulação, no tempo 12s, coloridas por velocidade. É possível observar, por exemplo, que para as Simulações 02, 05 e 06, que correspondem a um mesmo coeficiente de atrito, apresentaram melhor resultado quando comparadas ao valor experimental de ângulo de repouso para soja.

Também é possível observar uma região de maior velocidade próxima à parede do tambor rotativo, que decresce à medida que as partículas se aproximam do centro do tambor.

Tabela 3- Planejamento Composto Central para avaliação dos parâmetros.

\begin{tabular}{ccccll}
\hline Simulação & X1 & X2 & X3 & $\Phi\left({ }^{\circ}\right)$ & Erro $\left(^{\circ}\right)$ \\
\hline S1 & -1 & -1 & -1 & 52,79 & 1,94 \\
S2 & -1 & -1 & 1 & 41,12 & 3,10 \\
S3 & -1 & 1 & -1 & 65,97 & 2,42 \\
S4 & -1 & 1 & 1 & 65,49 & 4,03 \\
S5 & 1 & -1 & -1 & 41,47 & 3,54 \\
S6 & 1 & -1 & 1 & 40,04 & 2,91 \\
S7 & 1 & 1 & -1 & 71,36 & 2,68 \\
S8 & 1 & 1 & 1 & 68,58 & 1,19 \\
SS9 & $-1,575$ & 0 & 0 & 64,42 & 1,54 \\
S10 & 1,575 & 0 & 0 & 67,23 & 4,54 \\
S11 & 0 & $-1,575$ & 0 & 8,23 & 0,16 \\
S12 & 0 & 1,575 & 0 & 66,02 & 3,87 \\
S13 & 0 & 0 & $-1,575$ & 68,74 & 2,65 \\
S14 & 0 & 0 & 1,575 & 63,98 & 3,52 \\
S15 & 0 & 0 & 0 & 60,01 & 2,12 \\
S16 & 0 & 0 & 0 & 61,56 & 2,96 \\
S17 & 0 & 0 & 0 & 62,21 & 3,95 \\
S18 & 0 & 0 & 0 & 63,26 & 1,70 \\
S19 & 0 & 0 & 0 & 65,38 & 2,16 \\
S20 & 0 & 0 & 0 & 62,81 & 1,57 \\
S21 & 0 & 0 & 0 & 62,56 & 2,53 \\
\hline
\end{tabular}

Fonte: Autor (2015) 
Para que fossem identificados os parâmetros significativos no modelo de colisão molaamortecedor das partículas de soja, uma análise de regressão múltipla foi realizada.

Também foi feito um teste de hipótese $\mathrm{t}$ de Student e os coeficientes da equação estimados pelo método de mínimos quadrados, sendo que coeficientes com nível de significância maior que $15 \%$, $(\mathrm{p}>0,15)$ foram negligenciados. A Figura 5 apresenta a superfície de resposta obtida.
Figura 3 - Ângulo de repouso dinâmico da soja: (a) Experimental; (b) Melhor Simulação (S2).

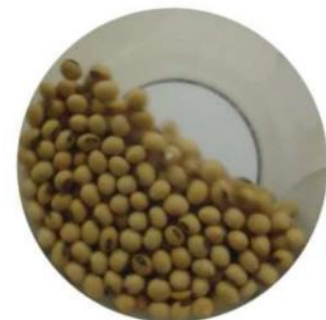

(a) $\left(41,4^{\circ}\right)$

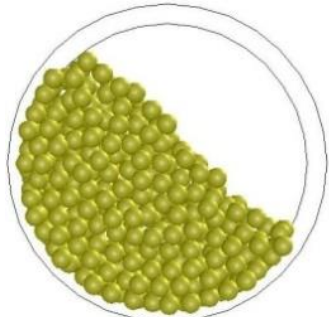

(b) $\left(41,1^{\circ}\right)$
Fonte: Autor (2015).

Figura 4 - Simulações DEM do tambor rotativo nas condições do PCC estudadas

$\mathrm{S} 1-52,8^{\circ}$

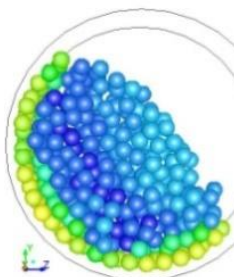

S5 - $41,5^{\circ}$
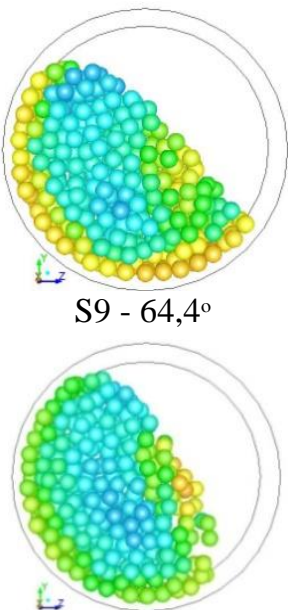

$\mathrm{S} 13-68,7^{\circ}$

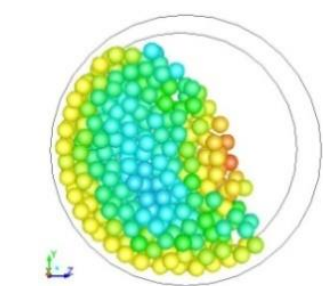

$2.10^{-1}$
S2 $-41,1^{\circ}$

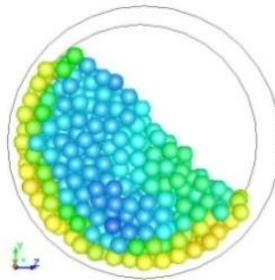

S6 - $40,0^{\circ}$

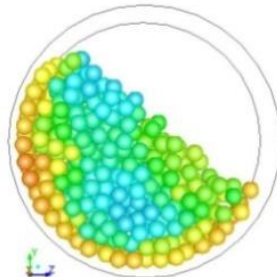

$\mathrm{S} 10-67,2^{\circ}$

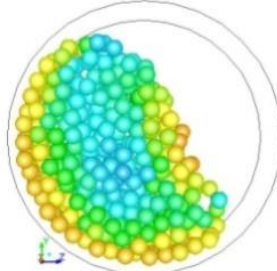

$\mathrm{S} 14-64,0^{\circ}$

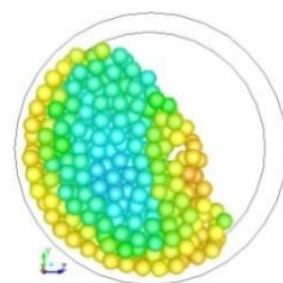

$\mathrm{S} 3-66,0^{\circ}$

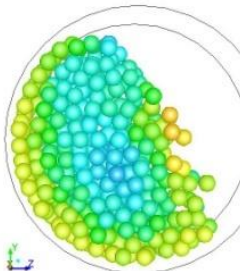

S7 $-71,4^{\circ}$

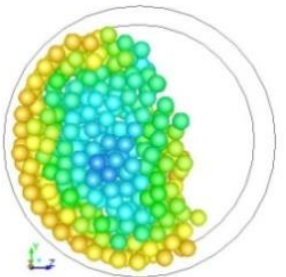

$\mathrm{S} 11-8,2^{\circ}$

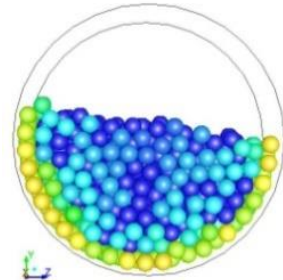

S15 - 60, $0^{\circ}$

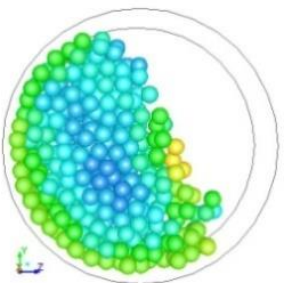

$\mathrm{S} 4-65,5^{\circ}$

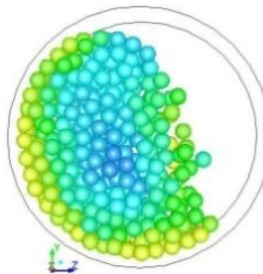

S8 - $68,6^{\circ}$

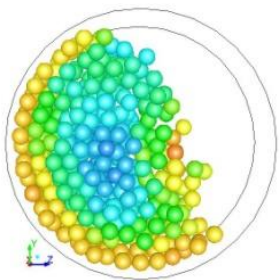

$\mathrm{S} 12-66,0^{\circ}$

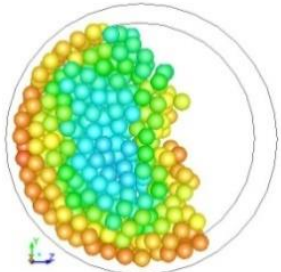

$\mathrm{S} 16-61,5^{\circ}$

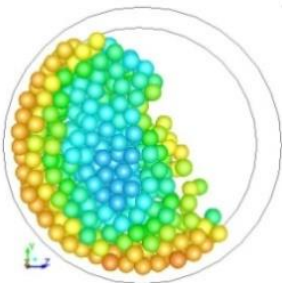

$1.10^{-3}$

$(\mathrm{m} / \mathrm{s})$

Fonte: Autor (2015). 
Figura 5 - Influência do coeficiente de elasticidade e coeficiente de atrito na resposta dos ângulos médios.

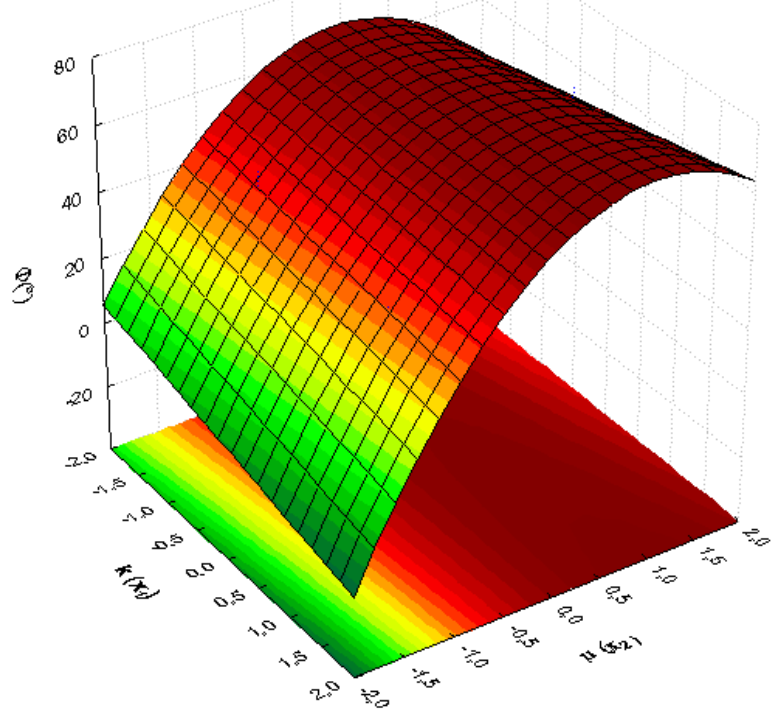

Fonte: Autor (2015).

O modelo reduzido do ângulo de repouso dinâmico simulado $\left(\Phi_{S}\right)$ é dado pela Equação $18\left(R^{2}=0,961\right)$ :

$$
\begin{aligned}
\Phi_{S}= & 64,466+14,430 X_{2}-10,074 X_{2}^{2}+ \\
& 2,615 X_{1} X_{2}
\end{aligned}
$$

em que $X_{1}$ representa o coeficiente de elasticidade (k) e $X_{2}$ o coeficiente de atrito $(\mu)$.

Através da análise estatística das simulações e da superfície de resposta, foi possível observar que, o parâmetro do modelo mola-amortecedor que mais influencia a resposta simulada do ângulo de repouso dinâmico é o coeficiente de atrito, tanto na forma linear quanto quadrática. A superfície de resposta dada pela Figura 5 mostra a interação entre o coeficiente de atrito e o de elasticidade, sendo notável que este segundo tem uma pequena influência sobre o resultado simulado.

\section{CONCLUSÕES}

Para o conhecimento da fluidodinâmica das partículas, é de suma importância a calibração dos parâmetros de colisão para cada material granular. A simulação dos ângulos de repouso é peça fundamental nesta calibração. A análise estatística dessas simulações permitiu observar que, para a semente de soja, o parâmetro do modelo mola-amortecedor que mais influencia a resposta simulada do ângulo de repouso dinâmico é o coeficiente de atrito. Quanto à comparação das simulações com o valor experimental, observa-se que as simulações 2 , 5 e 6 apresentaram resultados muito próximos ao experimental, mostrando que valores de coeficiente de atrito de 0,2 produzem os melhores resultados para a soja.

Dessa forma, indica-se usar: $400 \mathrm{~N} / \mathrm{m}$ para o coeficiente de elasticidade do choque; 0,2 para o coeficiente de atrito e 0,8 para o coeficiente de restituição.

\section{NOMENCLATURA}$$
p
$$$$
q
$$

$\alpha_{q}$

$\rho_{q}$

$\vec{v}_{q}$

$\nabla P$

$\overline{\bar{\tau}}_{q}$

$K_{p q}$

$\vec{v}_{p}$

$S_{\text {other }}$

$m$

$\vec{v}$

$\vec{F}_{\text {arrasto }}$

$\vec{F}_{\text {pressão }}$
Fase sólida

Fase fluida

Fração volumétrica da fase $\mathrm{q}$

Massa específica da fase $\mathrm{q}$

Velocidade da fase $\mathrm{q}$

Gradiente de pressão do fluido

Tensor tensão-deformação

Coeficiente de transferência de momento entre as fases $\mathrm{p} \mathrm{e} \mathrm{q}$

Velocidade da fase $p$

Outras formas de transferência de quantidade de movimento

Massa da partícula

Velocidade da partícula

Força de arrasto provocada pelo fluido

Força devido ao gradiente de pressão no fluido 
$\vec{F}_{\text {gravidade }}$ Força gravitacional

$\vec{F}_{\text {outras }} \quad$ Demais forças que atuam sobre a

partícula

$\Phi_{S} \quad$ Ângulo de repouso dinâmico

$\mathrm{X}_{1} \quad$ Coeficiente de Elasticidade

$\mathrm{X}_{2} \quad$ Coeficiente de Atrito

$\mathrm{X}_{3} \quad$ Coeficiente de Restituição

\section{REFERÊNCIAS}

CUNDALL P. A.; STRACK, O. D. L.. A discret numerical model for granular assemblies. Geotechnique. v. 29, p. 47-65, 1979.

CUNHA, Fabiano Guimarães. Estudo da Extração Mecânica de Bixina das Sementes de Urucum em Leito de Jorro. Dissertação (Mestrado em Engenharia Química) Universidade Federal de Uberlândia, Minas Gerais, 2008.

DI RENZO, A.; DI MAIO, F. P. Comparison of contact-force models for the simulation of collisions in DEM-based granular flow codes. Chemical Engineering Sci.v.59, p.525-541, 2004.

DUARTE, C. R. Estudo Experimental e de Simulação da Fluidodinâmica e Recobrimento em Leito de Jorro. Tese (Doutorado em Engenharia Química) Universidade Federal de Uberlândia, Uberlândia, 2006.

GAO, D., SUN, J. Using DEM in Particulate Flow Simulations. In Hydrodynamics Optimizing Methods and Tools, p. 29-50, 2011.

GRACE, J.R.; TAGHIPOUR, F. Verification and validation of CFD models and dynamic similarity for fluidized beds. Powder Technology. v. 139, p. 99-100, 2004.

JOHNSTONE, M.W. Calibration of DEM Models for granular materials using bulk physical tests. Teses (Doutorado em Filosofia)., University os Edinburgh, 2010.

PEREIRA, F.A.R. Escoamento Laminar de Líquidos Não-Newtonianos em Seções Anulares: Estudos de CFD e Abordagem
Experimental. Tese (Doutorado em Engenharia Química) - Universidade Federal de Uberlândia, Uberlândia, 2006.

SANTOS, K.G. Aspectos fundamentais da pirólise de biomassa em leito de jorro: Cinética e fluidodinâmica do processo. 2011. 235p. Tese (Doutorado em Engenharia Química) Universidade Federal de Uberlândia, Uberlândia, 2011.

SANTOS, K. G.; MURATA, V. V.; BARROZO, M. A. S. Three-dimensional computational fluid dynamics modeling of spouted bed. Can. J. Chem. Eng., v. 87, p. 211-219, 2009.

SOUZA, J. Z.; RIBEIRO, D. C.; FONTES, C. E.; LEIBSOHN, A.; WALDMANN, A.; LOMBA, R., Análise do Escoamento e Fluidos Particulados durante a Perfuração de Reservatórios Fraturados, III Encontro Nacional de Hidráulica de Poços, Campos do Jordão, São Paulo, p. 8, 2009.

SILVÉRIO, B. C. Estudos Fluidodinâmicos e de Secagem de Fertilizantes em Diferentes Tipos de Secadores Rotatórios. Tese (Doutorado em Engenharia Química) - Universidade Federal de Uberlândia, Uberlândia, 2012.

VIEIRA, L.G.M. Otimização dos Processos de Separação em Hidrociclones Filtrantes. 2006. Tese (Doutorado em Engenharia Química) Universidade Federal de Uberlândia, Uberlândia, 2006.

WACHS, A., GIROLAMI, L., VINAY, G., FERRER, G., Grains3D, a flexible DEM approach for particles of arbitrary convex shape - Part I: Numerical model and validations, Powder Technology, v. 224, p. 374-389, 2012.

WALTON, O. R., BRAUN, R. L. Simulation of a rotary drum and repose tests for friction spheres and rigid clusters. Workshop on Flow particulates and fluids, 1993.

ZHOU, Y.C. XU, B. H., YU, A.B., ZULLI, P., An experimental and numerical study of the angle of repose of coarse spheres, Powder Technology, v. 125 , p. 45- 54, 2002.

\section{AGRADECIMENTOS}

Os autores agradecem ao $\mathrm{CNPq}$ pela bolsa PIBIC de Iniciação Científica e à Capes. 